\title{
X-ray Emission from Single Stars
}

\author{
J. H. M. M. Schmitt \\ Hamburger Sternwarte, 21029 Hamburg, Gojenbergsweg 112, Germany
}

\begin{abstract}
During the last two decades a new field in stellar astrophysics emerged: Stellar X-ray astronomy. With the advent of soft X-ray imagery X-ray emission was found from many thousands of solar-like stars. I will summarize the most important X-ray properties of cool stars and how they compare to the Sun.
\end{abstract}

\section{Introduction}

The advent of X-ray imaging at soft X-ray wavelengths has led to the detection of X-ray emission from many thousands of stars similar to the Sun. This enormous increase in our knowledge of stellar X-ray emission has been derived almost exclusively from observations carried out with the Einstein Observatory, (operated between 1978 and 1981) and ROSAT (operated between 1990 and 1998). Coronal X-ray sources were also intensely studied at extreme ultraviolet wavelengths with the EUVE satellite operated between 1992 and 2000, and in particular, the first high spectral resolution observations of a larger sample for stars was performed with the spectrometers onboard EUVE. Stars were of course observed also with other X-ray satellites such as EXOSAT, ASCA and BeppoSAX, but the contributions from those missions were geared mostly to other topics.

X-ray emission is generally considered to be a key indicator of "magnetic activity", yet there is no general consensus or generally applied definition of solar and stellar activity. Usually one associates Sun spots, plage, flares, spicules and related phenomena with magnetic activity on the Sun and similar definitions apply for stars. Linsky (1985) defines solar-like (activity) phenomena as "nonradiative in character, of fundamentally magnetic origin and almost certainly due to a magnetic dynamo operating in or at the base of a convection zone." In magnetically active regions of the Sun and the stars one finds departures from pure radiative equilibrium caused by some kind of heating and probably momentum deposition processes. Linsky's (1985) definition is very useful because it provides a recipe for identifying activity through searching for evidence of non-radiative heating and showing its magnetic nature. Direct measurements of magnetic fields on other stars are possible but rather difficult. Direct measurements of coronal magnetic fields are very difficult even for the Sun and impossible for stars at present. However, it is straightforward to search for the heating effects associated with magnetic activity. Such evidence for non-radiative heating can be obtained by observations of the heated thermal plasma in the UV or X-ray domain or by observations of non-thermal emission from highly energetic particles 


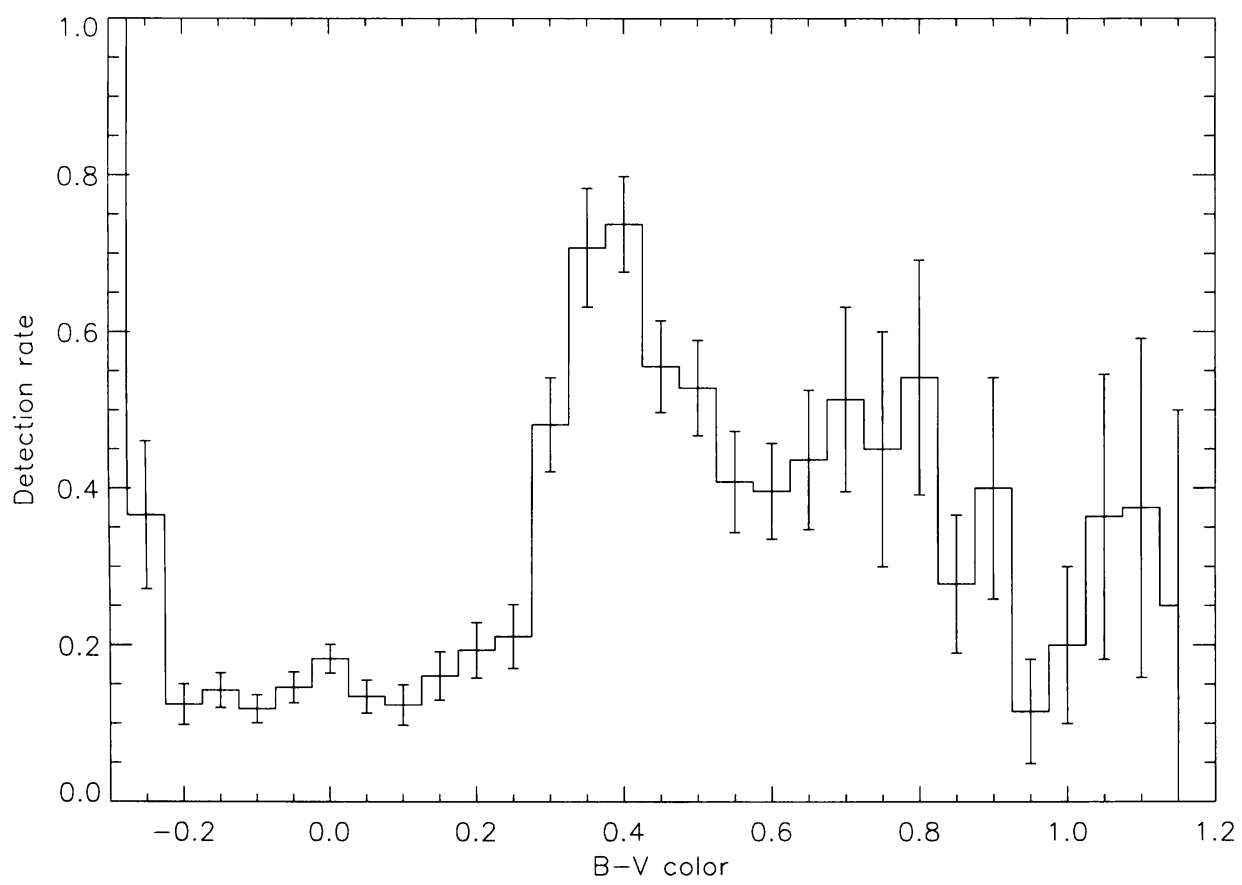

Figure 1. Detection rate for main sequence stars contained in the $\mathrm{BSC}$ catalog as a function of B-V color. Note the completeness of the detections among the early O-type stars and the large increase in detection rate at a B-V color of $\sim 0.3$. For large B-V colors the BSC catalog contains too few entries for meaningful discussion. In general, the detection rates for M-type dwarfs are high, while for M-type giants they are very small.

often accompanying and possibly intimately linked with the heating process(es). Further, such non-radiative heating is - usually - confined in space and time and therefore evidence for time variability or spatial structure is also good evidence for non-radiative heating. Thus the detection of quiescent and/or flaring X-ray emission from a star is indeed an excellent indicator of non-radiative heating.

\section{The Sun and the solar-stellar connection}

The high temperature of the solar corona - orders of magnitude hotter than the solar photosphere - had been known since the 1940's, when the mysterious coronal lines seen in the optical were identified as forbidden emission lines from highly ionized iron (Grottian 1939). The realization, however, that the solar corona represents a predominantly magnetic phenomenon came much later, in particular, when high-resolution solar X-ray images showed the coronal emission to come from a a great number of loop-like structures filled with hot plasma. Magnetic fields clearly had to play a key role both in structuring and heating the solar corona and one can view the solar corona as a prototype of a magnetically 
controlled thin plasma. This plasma does of course not exist on its own, but rather is linked to chromospheric and photospheric regions of activity. Since hot plasma loses the bulk of its energy in the X-ray band, X-ray emission is the prime method to search for and diagnose coronae around other stars. The specific advantage of $\mathrm{X}$-ray emission is due to the fact that it cannot be contaminated by photospheric emission even from the hottest non-degenerate stars; note in this context that only the X-ray emission from hot white dwarfs such as Sirius B or HZ 43 is "photospheric" in nature. On the other hand, chromospheric and transition region diagnostics at UV wavelengths are heavily affected by the photospheric flux from the parent star (depending on its precise spectral type) and therefore such observations are usually significantly biased in spectral type.

Within the framework of the ROSAT all-sky survey (RASS) it was possible to carry out a sensitive unbiased surveys of stellar X-ray emission. Of course, many stellar surveys were carried out with the previously available Einstein Observatory data, however, it is very difficult to show that these surveys are really unbiased. A typical figure for the sensitivity limit of the RASS is a limiting X-ray flux of $\approx 2 \times 10^{-13} \mathrm{erg} / \mathrm{cm}^{2} / \mathrm{sec}$, which implies that solar-like X-ray emission with X-ray luminosities of $\approx 2 \times 10^{27} \mathrm{erg} / \mathrm{sec}$ can be detected out to distances of $10 \mathrm{pc}$; in the ROSAT pointing program sensitivity increases of an order of magnitude with enusing increases in the detection distance can be easily reached. As a starting point, I investigate the X-ray properties of the brightest stars contained in the bright star catalog (BSC) and study their detection rate (in the RASS data) vs. spectral type. The BSC comprises all stars down to a magnitude of 6.5, i.e., around 10000 entries. Being a magnitude-limited catalog, its composition in terms of spectral type is biased towards intrinsically bright stars, and consequently it contains many stars of type A and F as well as giant stars. In Fig. $1 \mathrm{I}$ show the detection rate of main sequence stars listed in the $\mathrm{BSC}$ vs. B-V color. As is apparent from Fig. 1 , the detection rate is very large (essentially $100 \%$ ) for stars of spectral type O, it decreases among B- and A-type stars to between $10 \%-20 \%$. At spectral type F near the B-V-color $\approx 2$ the detection rate suddenly jumps to about $70 \%$, whereupon it decreases with error bars becoming larger and larger towards redder and redder colors. It is extremely suggestive to associate this sudden jump in detection rate with the "onset of convection", which is known to occur at that spectral type (cf., Schmitt et al. 1985). Somehow, the occurrence of X-ray emission seems to be linked to the interior property of a star. This finding coupled with the rotation-activity connection provides strong support for a picture that views magnetic activity as universal for all stars with outer convection zones (and rotation). The activity observed on the Sun would just be a special manifestation of the occurrence of magnetic activity in a cool star that happens to be located very close to us. With the huge amount of X-ray data now at our disposal we are in a position to compare solar X-ray properties with those observed for stars.

\section{X-ray emission from cool stars on the main sequence}

\subsection{Completeness of the RASS data}

The RASS was an essentially X-ray flux limited all-sky survey. Such surveys tend to find the intrinsically brightest objects of any given population since these are 


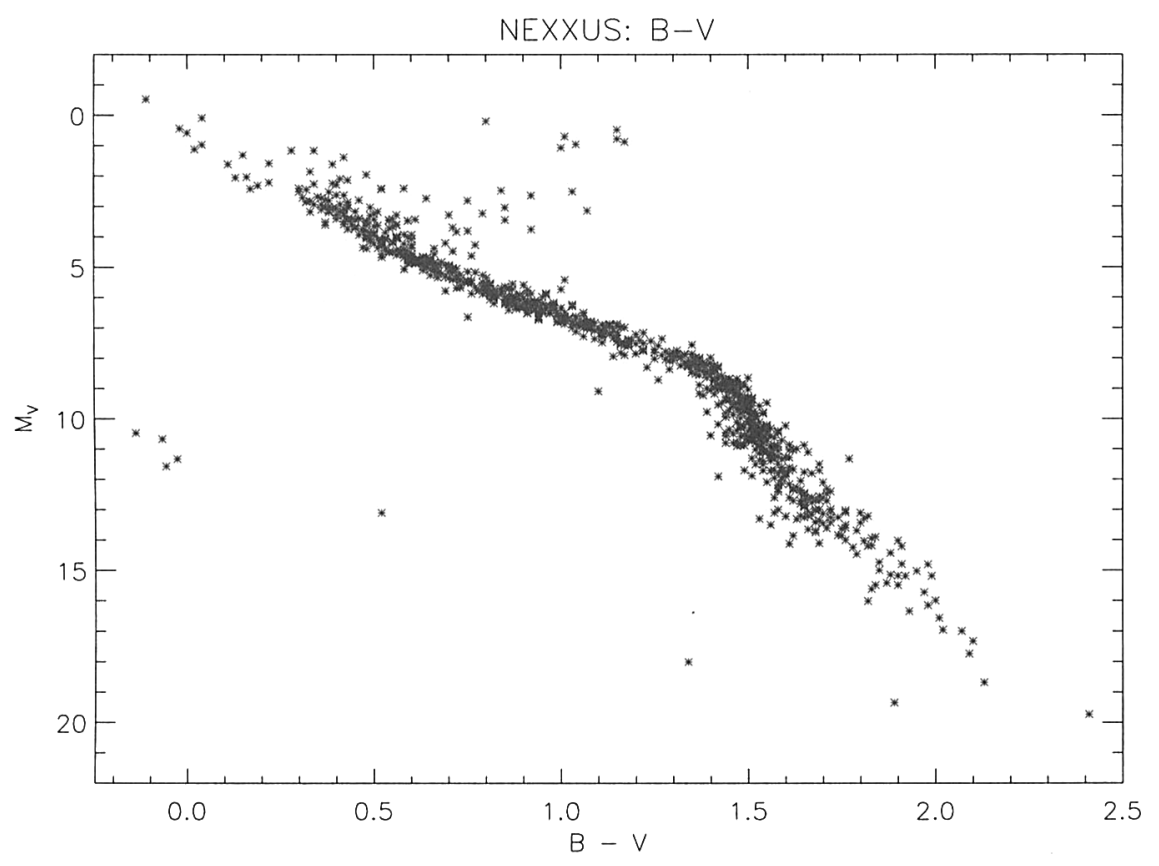

Figure 2. Color-magnitude diagram $\left(B-V\right.$ vs. $\left.M_{V}\right)$ for all X-ray detected stars listed in the NEXXUS data base.

sampled over the largest spatial volumes. Low-luminosity objects are sampled only over small spatial volumes and are therefore quite sparse in the RASS data. In order to study the X-ray properties of solar-like stars in an unbiased fashion and to compare the Sun to the stars in a fair way, one therefore has to consider volume-limited samples. Volume-limited samples with very large detection rates were constructed by Schmitt et al. (1995) for K and M dwarfs and Schmitt (1997) for F and G dwarfs by combining RASS data with ROSAT pointings at stars not detected in the RASS. Schmitt \& Liefke (2003) summarize the ROSAT observations by including all available ROSAT data and using the HIPPARCOS distance scale. Schmitt \& Liefke (2003) searched the ROSAT source lists and the CNS4 catalog for positional coincidences using a matching criterion of 120 arcsec for survey data, 60 arcsec for pointing data with the ROSAT PSPC and 30 arcsec for ROSAT HRI data. Altogether 1333 of the 3231 stars (41.1\%) up to a distance of $25 \mathrm{pc}$ contained in the CNS4 catalog can be associated with ROSAT detected X-ray sources. The vast majority of these sources (1217) comes from the RASS data, while the remaining 116 stars were only detected in pointed observations. In addition to the survey detections, 328 stars were also observed with the PSPC in pointed mode without the boron filter, 49 with the filter, and 242 stars were observed with the ROSAT HRI. Moreover, some of these stars were observed more than once, so that multiple detections of coronal X-ray emission are available. For easy access and future reference the results of this cross-correlation process were assembled in the Nearby X-ray and XUV-emitting Stars data 
base, available via www from the Home Page of the Hamburger Sternwarte at the URL http://www.hs.uni-hamburg.de/DE/For/Gal/Xgroup/nexxus. A colormagnitude diagram of all NEXXUS stars is shown in Fig. 2. One immediately notes the well-known paucity of X-ray emitting white dwarfs as well as the absence of X-ray detected brighter giants. Stellar X-ray emission is detected down to an absolute magnitude of $M_{V}=20$, i.e., down to the very bottom of the main sequence.

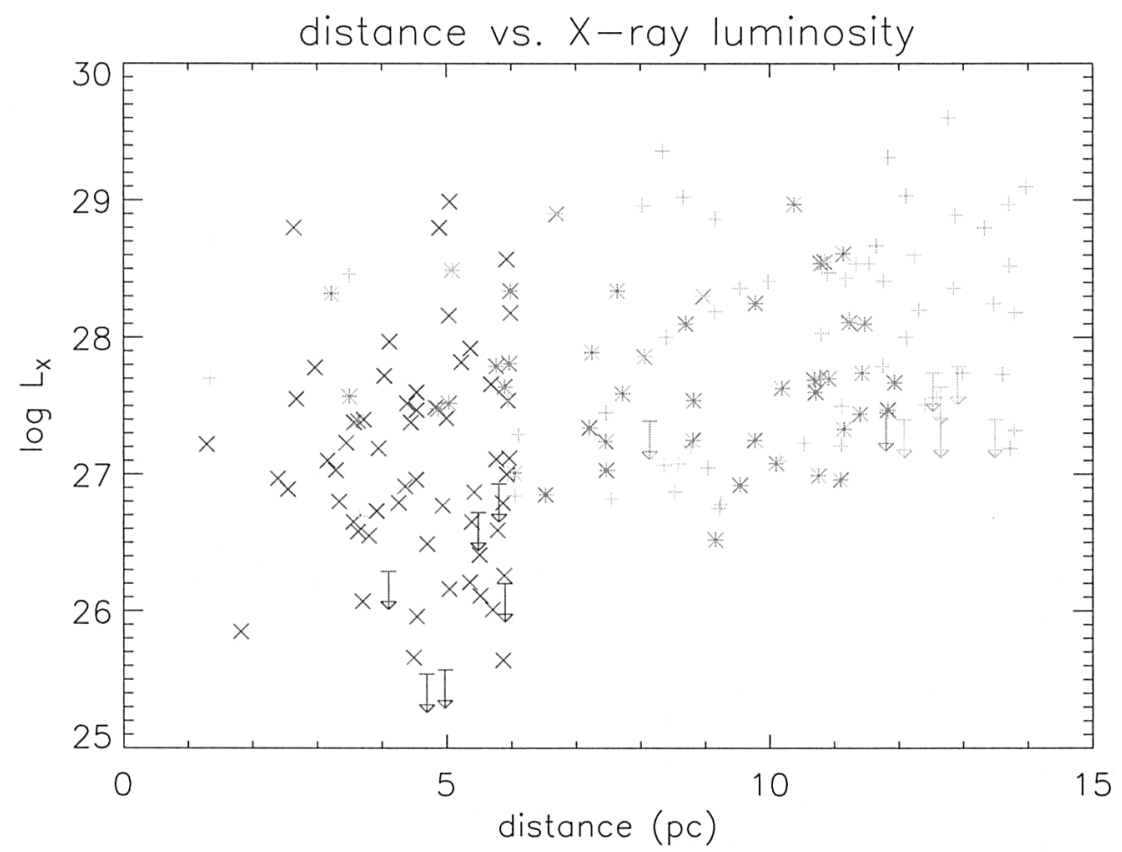

Figure 3. X-ray luminosity vs. distance for nearby cool stars. Note the absence of any distance-dependent bias in the X-ray luminosities and detection rates of cool stars. X's denote M-type stars, star symbols mark K-type stars, and plus symbols represent F-type stars.

\subsection{The nearby cool stars}

How complete are now the detections in the NEXXUS data base ? Defining F/Gstars to be those with absolute magnitudes $M_{V}$ in the range $3 \leq M_{V} \leq 5.80, \mathrm{~K}$ stars those with $5.80<M_{V} \leq 8.50$, and as M stars those fainter than $M_{V}=$ 8.50 we plot (in Fig. 3) for these stars X-ray luminosity $L_{X}$ as a function of distance. Among $69 \mathrm{~F} / \mathrm{G}$ stars within a distance of $14 \mathrm{pc}$ around the Sun only seven remain undetected, i.e, detection rate within the volume out to $14 \mathrm{pc}$ is therefore $94 \%$, and all stars within $12 \mathrm{pc}$ have been detected. Out of $51 \mathrm{~K}$ stars within $12 \mathrm{pc}$ only two stars have not been detected and hence the detection rate is $96 \%$, while out of $65 \mathrm{M}$ stars within $6 \mathrm{pc} 6$ stars have not been detected. Most of the non-detected M-type stars are brown dwarfs or very low-mass stars; the only nearby M star not detected in a short PSPC pointing is GJ 1002 and all 
stars within $4 \mathrm{pc}$ have been detected. The conclusion from this exercise obviously is that the detection rate among the nearest stars is considerably larger than $41.1 \%$ and in fact there is no reason to expect that these stars will not be detected once more sensitive X-ray observations are available. In other words, the formation of X-ray emitting coronae appears to be universal for late-type main sequence stars. A corona containing hot plasma $\left(\mathrm{T}>10^{6} \mathrm{~K}\right)$ is always formed at the interface between a turbulent outer convection zone and space, and X-ray dark solar-like stars do not exist (at least within the immediate solar environment).
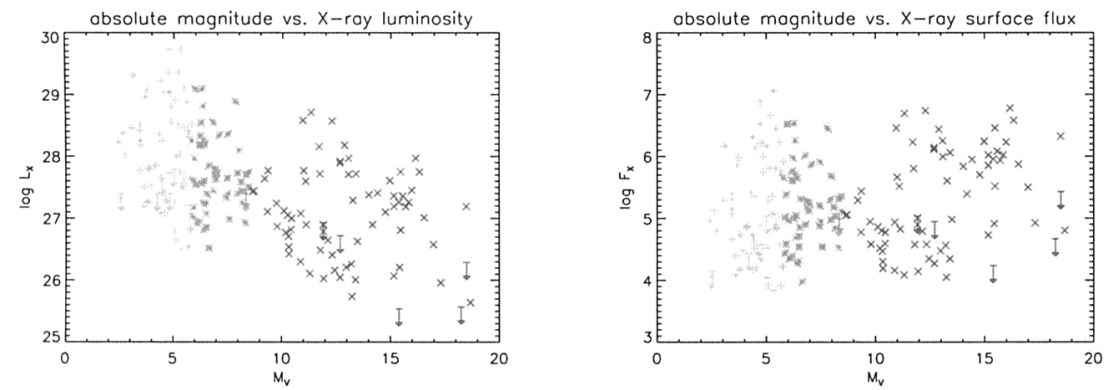

Figure 4. Left Panel: X-ray luminosity vs. B-V colors for nearby cool stars. Right Panel: X-ray surface flux vs. B-V color for nearby cool stars; meaning of symbols as in Fig. 3.

It is interesting to consider the X-ray luminosities of the so-defined sample of cool stars; given that HIPPARCOS parallaxes are known to all those stars, the conversion from count rate to flux is now the main uncertainty in the derived $\mathrm{X}$-ray luminosities. In Fig. 4 we plot the X-ray luminosities for the above defined F/G-type stars (within $14 \mathrm{pc}$ ), the K-stars (within $12 \mathrm{pc}$ ) and the Mstars (within $6 \mathrm{cp}$ ). As is well known, thare is a large spread in X-ray luminosity from stars to star with a decreasing trend towards later types. This trend can be taken out by considering the mean X-ray surface fluxes, which are plotted vs. B-V-color in Fig. 5. One notices in Fig. 5 the existence of a rather well defined lower envelope $F_{\text {lim }}$ to the observed mean X-ray surface flux distribution. The apparent cutoff at surface fluxes of $F_{\text {lim }} \approx 10^{4} \mathrm{erg} / \mathrm{cm}^{2} / \mathrm{sec}$ is clearly not a question of lacking sensitivity since the non-detected A-type stars do indeed have upper limits below $F_{\text {lim }}$. Because of the samples' completeness properties both for the $\mathrm{F}$ and $\mathrm{G}$ stars as well as the $\mathrm{K}$ and $\mathrm{M}$ stars, we can therefore state that among cool dwarfs stars with X-ray surface fluxes below $F_{\text {lim }}$ do not exist (in the considered volumes of space). The lower limit to the X-ray surface flux actually compares well with the observed X-ray surface flux from solar coronal holes which lies at levels of $\approx 10^{4} \mathrm{erg} / \mathrm{cm}^{2} / \mathrm{sec}$ and it is suggestive to interpret the stars observed at their minimum flux levels as stars surrounded by coronal holes without any active regions.

\subsection{A-type stars}

According to stellar structure theory A-type stars are devoid of any outer convective zones and should hence be devoid of any coronal emission. Yet the 
detection rate of A-type stars in the RASS data is about $15 \%$ (cf., Fig. 1). On the other hand, X-ray emission from the prototypical narby star Vega could not be detected, and the obtained upper limit of $1.210^{-3} \mathrm{cts} / \mathrm{sec}$, which in fact is attributed by Schmitt (1997) to UV contamination, would place Vega - if interpreted as true X-ray flux - with an X-ray luminosity of $L_{X} \sim 5.510^{25} \mathrm{erg} / \mathrm{sec}$ at the very bottom of all cool stars. Clearly the physics of coronal formation in those stars - if coronae exist at all - must be very different and in all likelihood stars like Vega do not possess any corona. The standard hypothesis to explain the observed X-ray emission from (some) A-type stars is to attribute the X-ray emission to optically fainter companions; obviously, M-dwarfs can be rather easily "hidden" around a bright A-type star. In a few cases this hypothesis can be directly tested. For example, Chandra observations of the A star visual binary $\alpha$ Gem (Stelzer \& Burwitz 2003) show X-ray emission from both components, which in turn are known to be spectroscopic binaries. In another special case of the eclipsing binary $\alpha \mathrm{CrB}$ the late-type secondary (spectral type G5V) is totally occulted by the early-type primary (spectral type A0V). Schmitt \& Kürster (1993) were the first to report a total X-ray eclipse at the time of optical secondary minimum; further total eclipses were studied by Schmitt (1998) and Güdel et al. (2003), leading to an upper limit of about $6 \times 10^{26} \mathrm{erg} / \mathrm{sec}$ for the X-ray luminosity of $\alpha \mathrm{CrB}$.
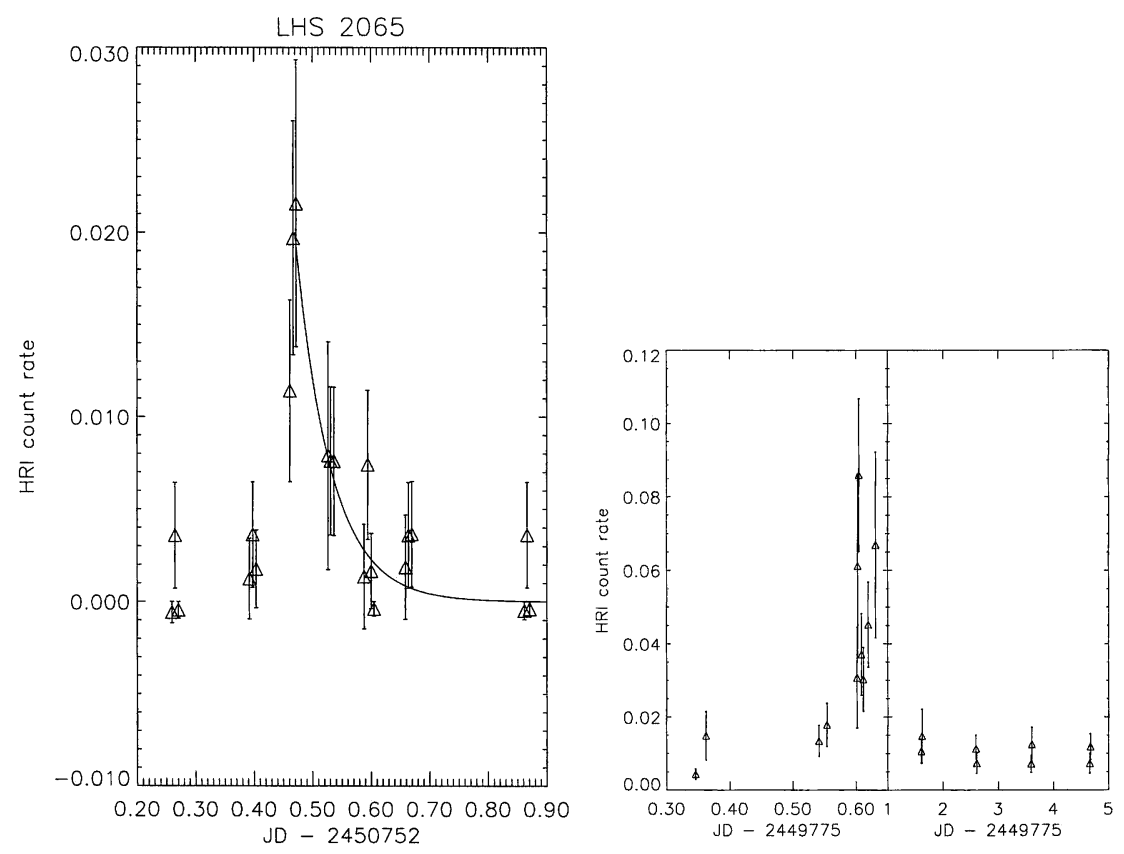

Figure 5. Left Panel: ROSAT HRI of a strong flare on the late-type M star LHS 2065; Right panel: ROSAT HRI light curve of the peculiar B-type star $\sigma$ Ori E; note the break between the two parts of the figure. 


\section{X-ray emission at the bottom of the main sequence}

According to the above described paradigm of stellar activity one needs both convection and rotation as necessary ingredients for the observed plethora of activity phenomena. Just as the onset of convection for late A/early $F$ stars (cf., Fig. 1) is of importance for dynamo theory, so is the very bottom of the main sequence. According to the stellar structure theory stars of spectral type dM3 or later, or in terms of mass, stars $\leq 0.3 M_{\odot}$, are expected to be become fully convective, and should therefore not have an interface between an interior radiative and outer convective zone. Interestingly, the observed X-ray properties of M-dwarfs do not change for such fully convective dwarfs. Considering the ratio $L_{X} / L_{b o l}$, i.e., the efficiency, with which convective flux is eventually converted into X-ray flux, one does not find any evidence for any change neither for very low mass stars (Fleming et al. 1993), nor for field stars (Schmitt et al. 1995). For the X-ray properties of a main-sequence stars it thus appears to be irrelevant whether the star's interior is fully convective or not.

While it is true that one of the latest known star, LHS 2924, could not be detected by Fleming et al. (1993), as well as BRI 0021-0214 (Neuhäuser et al. 1999), other rather similar stars have been detected (i.e., LHS 3003, cf. Schmitt et al. 1995), suggesting that the apparent lack of detections of very low mass stars is due only to insufficient sensitivity given the the rather large distance of these objects; for LHS 2924 one finds an upper limit of only $L_{x} / L_{b o l}<4.510^{-5}$ ). In fact, X-raX-ray emission has even been detected for a few brown dwarfs, i.e., in objects below the hydrogen burning limit $\left(\sim 0.085 \mathrm{M}_{\odot}\right)$, but almost all of these objects are young and therefore have "large" surface temperatures and early spectral types. Interestingly, flaring X-ray emission has been detected with ROSAT from the nearby very late-type stars vB10 ( (=Gl752B; Fleming et al. 2000) and LHS 2065 (Schmitt \& Liefke 2002), and further evidence for large X-ray flares in very low-mass stars has been provided by Schmitt \& Liefke (2003) for LHS 288 and by Rutledge et al. (2000) for the brown dwarf LP944-20. Thus we conclude that X-ray emission is likely to occur for all stars down to the bottom of the main sequence; for the very low-mass stars and the brown dwarfs $\mathrm{X}$-ray emission may actually occur only in a transient fashion.

\section{X-ray emission from early-type stars: A magnetic origin ?}

Fig. 1 clearly demonstrates that X-ray emission is also very common for earlytype stars of spectral type $\mathrm{O}$ and $\mathrm{B}$. The $\mathrm{X}$-ray emission from these stars is thought to originate from instabilities in their radiatively driven winds, rather from magnetic effects. This view is supported by a number of findings: (a) The X-ray temperatures of early-type stars are far lower than those of "active" cool stars. (b) The efficiency of X-ray production measured in terms of $L_{X} / L_{b o l}$ is far lower than the value found in fact for almost any cool star. (c) X-ray variability is only rarely found among early-type stars.

One problem in this picture is the explanation of X-ray emission from Aand B-type stars, which should have either weak winds or no winds to produce any significant X-ray emission. The X-ray emission observed from these stars is then interpreted as X-ray emission from optically faint, late-type companions, 
which can always be easily hidden around a bright main sequence star. No real counter example, that would in fact contradict this interpretation, can be provided (absence of evidence is not evidence of absence), however, a number of observations have been reported, which actually suggest magnetic-field related activity in early-type stars. While in general X-ray variability in early-type star is not common, precisely such variability has been reported in two early-type stars with measured magnetic fields. Gagné et al. (1997) report a periodic variation in the $\mathrm{X}$-ray flux of $\theta^{1}$ Ori $\mathrm{C}$, which appears to be modulated with the star's rotation period of 15.4 days, implying, that the X-ray emission cannot be uniformly distributed in the wind of $\theta^{1}$ Ori C. Furthermore, an X-ray flare on the peculiar star $\sigma$ Ori $\mathrm{E}$, which again has a measured magnetic field in excess of 10 kG, has been reported by Groote \& Schmitt (2003); XMM-Newton observations of the same object reported by Pallavicini et al. (2003) demonstrate that such flares seem to occur quite frequently. While Pallavicini et al. (2003) argue that the flare observed by XMM-Newton occurs on a hitherto unseen late-type companion, Groote \& Schmitt (2003) present a variety of arguments suggesting the B-type star as the actual flare site and describe a physical scenario for an explanation of the flaring X-ray emission. These two observational findings of Xray variability on early-type stars with known magnetic fields are very intriguing and may in fact point at a closer than previously assumed relationship between the X-ray emission from early- and late-type stars.

\section{X-ray emission off the main sequence}

Just like on the main-sequence, X-ray emission for giants to the left of the dividing line seems to be ubiquitous. In the study of a complete volume-limited sample of giants within 25 pc around the Sun using both ROSAT survey and pointing data Hünsch et al. (1996) found that all stars, which remained undetected in the survey data but observed in the pointing program with sufficient sensitivity to detect solar-like emission levels, were in fact detected, a finding, which led to the conclusion that giants to the left of the XDL, which all have outer convection zones, are also ubiquitous X-ray emitters. For giants the XDL occurs across a very narrow region in the $\mathrm{H}-\mathrm{R}$ diagram. Thus a $\mathrm{G}$ giant (to the left of the XDL) can have a rather high X-ray luminosity of up to $L_{X} \sim 3 \times 10^{30}$ ergs $s^{-1}$, although such objects seem to be relatively rare while $\mathrm{K}$ giants to the right of the XDL can be almost five orders of magnitude fainter. For example, Ayres, Fleming and Schmitt (1991) were not able to detect the nearby giant Arcturus and obtained an upper limit of $L_{X}<3 \times 10^{25} \mathrm{ergs} \mathrm{s}^{-1}$; expressing this upper limit in terms of mean X-ray surface flux, this X-ray non-detection is more than 1000 times fainter than a solar coronal hole (cf., Fig. 4). The concept of a dividing line seems to disappear, however, among the brighter giants and supergiants. Among those stars there is a group of stars exhibiting both signatures of transition region material (as inferred from CIV line detections) as well as cool winds (inferred from UV line profiles), i.e., the so-called hybrid stars. As a result of extensive ROSAT observations hybrid stars are now known to possess hot coronal plasma at temperatures in the $10^{6}-10^{7} \mathrm{~K}$ range (Haisch, Schmitt \& Rosso 1992; Reimers \& Schmitt 1992; Kashyap et al. 1994; Reimers et al. 1996). 
The observational situation is summarized in Fig. 6, where the stars reported by Hünsch et al. (1996) within 25 pc around the Sun and the hybrid stars discussed by Reimers et al. (1996) are shown in an HR-diagram. The nearby giants detected as X-ray sources are plotted as dark gray circles, those not detected as X-ray sources as white circles, the X-ray detected hybrid stars are shown as black circles and the hybrid stars not detected as X-ray sources as light grey circles. As is obvious from Fig. 6, the XDL shows very clearly up for giants of luminosity class III, while most of the hybrid stars have been detected as X-ray sources, some of them having a spectral type which puts them well beyond the XDL for luminosity class II giants. The general concept of a dividing line has been questioned by Hünsch and Schröder (1996), who propose a somewhat different scenario. They plot the X-ray detected giants and hybrid stars in a Hertzsprung-Russell diagram and note that all X-ray detections lie to the left of an evolutionary track with $M=1.25 M_{\odot}$. Thus in their scenario stars never actually cross the XDL, rather the XDL is interpreted as an effect of stellar evolution, since low-mass stars ascending the giant branch are restricted to a rather narrow mass range and must therefore be rather rare.

X-ray emission from M giants is extremely rare. Hünsch et al. (1998) identified about a dozen of candidates for X-ray emitting $\mathrm{M}$ giants. Using the high angular resolution of the Chandra telescope they were able to confirm the $\mathrm{X}$-ray emission from these objects and in particular show that the X-ray emission does indeed come from the $\mathrm{M}$ giant stars, rather than an coincidental nearby object. Since most of these $\mathrm{M}$ giants must be old, the extremely large X-ray luminosities in excess of $10^{30} \mathrm{erg} / \mathrm{sec}$ are difficult to explain. Hünsch et al. (2003) give a detailed account of the current status of X-ray emitting M giants, which represent a true puzzle for our understanding of the evolution of stellar activity.

\section{References}

Ayres, T.R., Fleming, T.A., \& Schmitt,J.H.M.M., 1991, ApJL, 376, L45.

Fleming, T.A., Giampapa, M.S., Schmitt, J.H.M.M., \& Bookbinder, J.A., 1993, ApJ, 410, 387.

Fleming, T. A., Giampapa, M. S., \& Schmitt, J. H. M. M., 2000, ApJ, 533, 372

Gagné, M., Caillault, J.-P., Stauffer, J., \& Linsky J.L., 1997, ApJ, 478, L78

Groote, D., \& Schmitt, J.H.M.M., 2003, A\&A, submitted

Grottian, W., 1939, Naturwiss., 27, 214.

Güdel, M., Arzner, K., Audard, M., \& Mewe, R., 2003, A\&A, 403, 155

Haisch, B., Schmitt, J.H.M.M., \& Rosso, C., 1992, ApJL, 388, L61.

Hünsch, M., Schmitt, J. H. M. M., Schröder, K.-P., \& Reimers, D., 1996, A\&A , 310, 801 .

Hünsch, M., \& Schröder, K.-P., 1996, A\&A 309, L51

Hünsch, M., Schmitt, J.H.M.M., \& Voges, W.H., 1997, A\&A Supp, 127, 251

Hünsch, M., Schmitt, J. H. M. M., Schröder, K., \& Zickgraf, F., A\&A, 330, 225

Hünsch, M., Konstantinova-Antova, R., de Medeiros, J., Kolev, D., \& Schmitt, J. H., IAU Symposium, 203 
Kashyap, V., Rosner, R., Harnden, F.R. Jr., Maggio, A., Micela, G., \& Sciortino, S., 1994, ApJ, 431, 402.

Linsky, J. L., 1985, Solar Physics, 100, 333

Neuhäuser, R., Briceńo, C., Comerón, F., Hearty, T., Martiacuten, E. L., Schmitt, J. H. M. M., Stelzer, B., Supper, R., Voges, W., \& Zinnecker, H., A\&A, 1999, 343, 883

Neuhäuser, R., \& Comerón, F., Science, 1998, 282, 83

Pallavicini R., Sanz-Forcada J., \& Franciosini E., 2002, in "High Resolution X-ray Spectroscopy with XMM-Newton \& Chandra", Proceedings of the international workshop held at the Mullard Space Science Laboratory of University College London, Holmbury St Mary, Dorking, Surrey, UK, October 24 - 25, 2002, Ed. Branduardi-Raymont, G., published electronically and to be stored on CD., p. E29

Reimers, D., \& Schmitt, J.H.M.M., 1992, ApJL, 392, L55.

Reimers, D., Hünsch, M., Schmitt, J.H.M.M., \& Toussaint, 1996, A\&A , 310, 801.

Rutledge, R. E., Basri, G., Martín, E. L., \& Bildsten, L., 2000, ApJ, 538, L141

Schmitt, J.H.M.M., Golub, L., \& Harnden, F.R. Jr., Maxson, C.W., Rosner, R., \& Vaiana, G.S., 1985, ApJ , 290 , 307.

Schmitt, J.H.M.M., Fleming, T.A., \& Giampapa, M.S., 1995, ApJ, 450, 392

Schmitt, J.H.M.M., 1997, A\&A, 318, 215

Schmitt, J.H.M.M., \& Liefke, C., 2002, A\&A, 382, L9

Schmitt, J.H.M.M.,\& Liefke, C., 2003, A\&A, in press

Stelzer, B., \& Burwitz, V., 2003, A\&A, 402, 719 


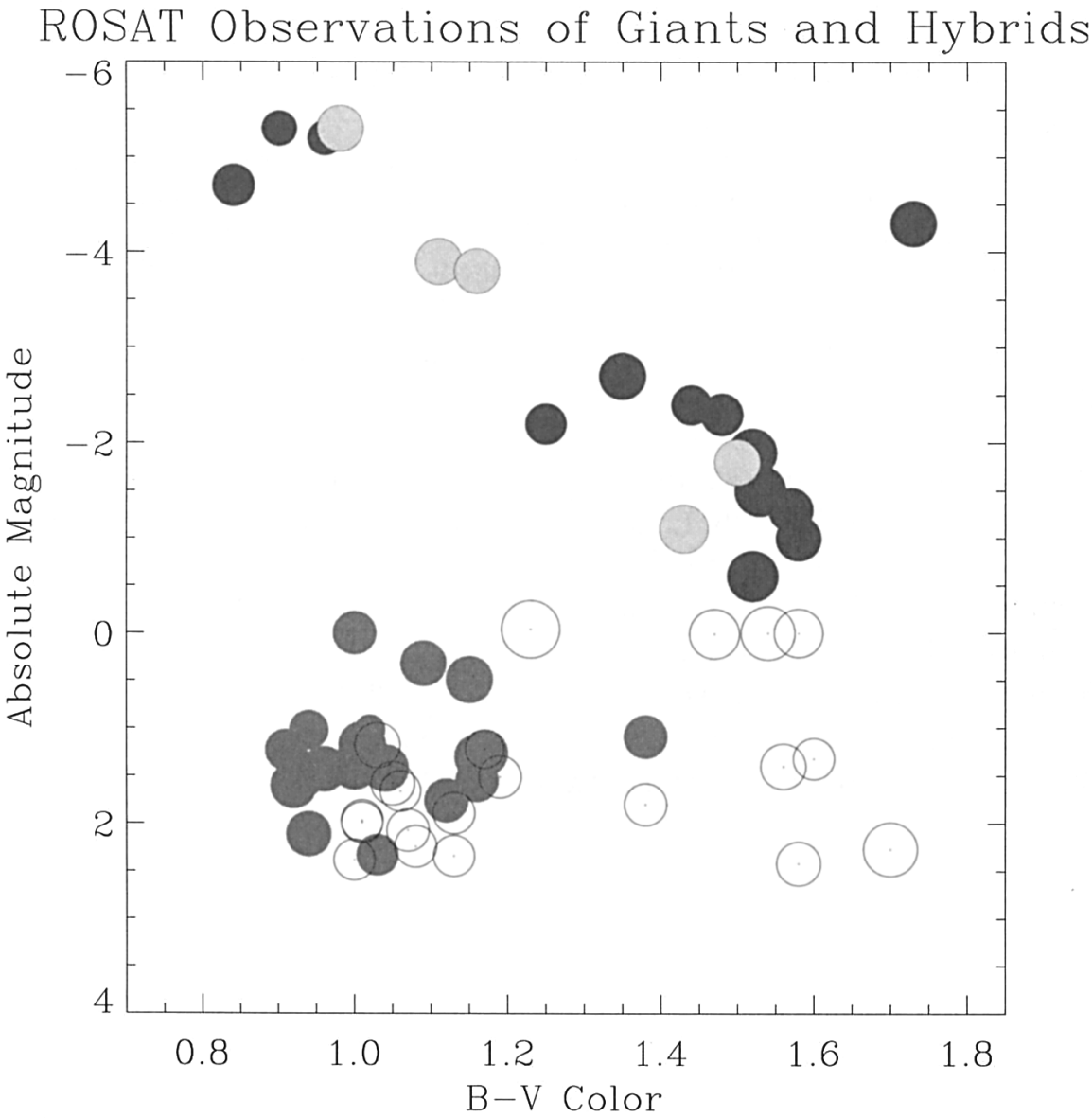

Figure 6. X-ray "bubblegram" of a complete sample of giants within $25 \mathrm{pc}$ around the Sun (from Hünsch et al. 1996) and hybrid stars (discussed by Reimers et al. 1996). Plotted are the HR-diagram positions of nearby giants detected as X-ray sources (dark gray circles), the positions of nearby giants not detected as X-ray sources (white circles), the positions of hybrid stars detected as X-ray sources (black circles) and those of hybrid stars not detected as X-ray sources (light grey circles). 